\title{
Shaking during Ion-Atom Collisions
}

\author{
P. Sharma* AND T. NANDI \\ Inter University Accelerator Centre, Aruna Asaf Ali Marg, New Delhi 110067, India \\ (Received June 10, 2015; in final form May 24, 2016)
}

\begin{abstract}
The shaking (shakeup and shakeoff) processes accompanying ion-atom collisions are studied using nonrelativistic hydrogenic wave functions for the $K-, L$ - and $M$-shell electrons in the sudden approximation limit. The role of recoil amplitude in the shaking processes is discussed. It is found that the suddenness of collision between projectile and target nuclei plays a more definitive factor in the shaking of the respective atomic system than the recoil of nuclei.
\end{abstract}

DOI: 10.12693/APhysPolA.130.697

PACS/topics: 31.15.xp, 34.50.Fa, 25.70.Bc

\section{Introduction}

During the various nuclear and atomic interactions, corresponding atomic system goes through the successive or an abrupt change in the central potential and/or electronic environment. If the perturbation occurs suddenly enough in the atomic system, then the orbital electrons may not react so rapidly to rearrange themselves. This process subsequently leads to the electrons getting excited to an unoccupied bound state, i.e. shakeup process or/and leaving the parent system, i.e. shakeoff process [1]. Collectively, these processes are called shaking process [2]. Theoretically, shaking process is treated under the sudden approximation limit [3]. It is worth to note that a perturbation is called sudden if the time period $\tau$ of perturbation is less than that of periodic motion, $2 \pi \omega_{n}^{-1}$, of the orbital electrons [4]. Interestingly, the condition of suddenness satisfies during various nuclear processes e.g. $\beta$-decay $[5,6], \alpha$-transfer reactions [7], positron decay [8], internal conversion [9, 10], orbital electron capture $[11,12]$ as well as in the atomic processes e.g. photoionization [13], inner-shell ionization [14], etc. In a similar phenomenon, if the atomic nucleus receives a sudden jolt in its normal state, it may lead to the ionization or excitation of electrons in the atom. During the fast ion-atom collisions target or projectile nucleus receives a similar type of sudden jolt, which can result in a sudden change of the position of the respective nucleus. Subsequently, this creates a sudden perturbation in the atomic electronic configuration, which initiates the shaking process. The sudden perturbation in the electronic environment solely depends on the recoil of the target atom or incident projectile ion and therefore, it affects both the atomic systems in the same manner. Using non-relativistic hydrogenic wave functions, the basic formulation to calculate the shaking probability during the sudden jolt of nuclei is described in the book by Landau and Lifshitz [15]. However, the work is only limited to the hydrogen atom case. In the present work, we have

\footnotetext{
${ }^{*}$ corresponding author; e-mail: prashant@iuac.res.in
}

extended the previous work and defined the general expressions for shaking probability of recoiling nuclei for $K$-, $L$ - and $M$-shell electrons of corresponding hydrogenlike atomic system.

\section{Formalism of the shaking process}

Basically, shaking process is a two-step process. In the first step, the central potential or electronic environment of parent atomic system gets disturbed due to the sudden perturbation, while in the second step under such influence electrons get excited to a new bound state (shakeup) or get ionized (shakeoff). In the case of ion-atom collisions, the first step correlates with the sudden impact of the projectile nuclei to the target nuclei. Whereas, the second step corresponds to the initiation of the shaking process. Worth to note that shaking due to the sudden jolt of nuclei strongly depends on the first step i.e the ion-atom recoil amplitude, whereas shaking due to other processes e.g. inner shell ionization [14], photoionization [13] etc. does not depend on the physical nature of the first step.

From the general quantum mechanics, the shaking probability of the atomic system can be determined by the overlap integral of the wave functions of the corresponding states [15-17]:

$$
W_{\mathrm{fi}}=\left|\int \psi_{\mathrm{f}}^{*} \psi_{\mathrm{i}}^{(0)} \mathrm{d} q\right|^{2} .
$$

Here, $\psi_{\mathrm{i}}^{(0)}$ and $\psi_{\mathrm{f}}$ are the stationary wave functions corresponding to the initial state of the atomic system with the unperturbed Hamiltonian $\left(\widehat{H}_{0}\right)$ and final state of the atomic system with the perturbed Hamiltonian $(\widehat{H})$, respectively. Due to the stationary nature, the wave functions associated with the probability density possess no time dependence and represent the form $\Psi=\psi(q) \exp (-\mathrm{i} E t / \hbar)$, where the wave functions are independent of the energy and solely depend on the position coordinates. As a consequence of the sudden perturbation, the electronic wave functions of the atomic system have no time to change from initial to final states and therefore remain the same as they were before the perturbation. However, they will no longer represent the characteristic wave functions of the new Hamiltonian of 
the system and thus, do not correspond to the stationary states. In other words, shaking phenomenon is the imperfect overlap of the initial and final electronic wave functions. Using non-relativistic hydrogenic wave functions, Eq. (1) can be rewritten for hydrogen-like atomic system as follows:

$$
W_{\mathrm{fi}}=\left|\int \psi_{n^{\prime} l^{\prime} m^{\prime}}^{*} \psi_{n l m} \mathrm{~d} q\right|^{2},
$$

where $\psi_{n^{\prime} l^{\prime} m^{\prime}}$ and $\psi_{n l m}$ are the initial and final state wave functions corresponding to $n, l, m$ and $n^{\prime}, l^{\prime}, m^{\prime}$ quantum numbers, respectively. Furthermore, the shake process has a monopole character which only favours such electronic transitions in which the principal quantum number of the final state is different from the initial state i.e. $n^{\prime} \neq n$ and all other quantum numbers remain the same. Thus, Eq. (2) can be simplified as follows:

$$
W_{\mathrm{fi}}=\left|\int \psi_{n^{\prime} l m}^{*} \psi_{n l m} \mathrm{~d} q\right|^{2} .
$$

\section{Theoretical work, results and discussion}

It is worth to mention that after the sudden change in the central potential or electronic environment, each electron has three possibilities in the new environment. It may remain either in the same state or can make a transition to unoccupied bound state (shakeup) or gets ionized to the continuum state (shakeoff). It is already mentioned in the previous section that shaking of atom/ion is the sum of shakeup and shakeoff processes. So in the other way, shaking probability can be calculated by subtracting the probability that all the electrons will remain in the same initial state from the total probability i.e. unity. This method was also applied by the earlier workers $[14,18]$ to calculate the shaking probabilities following the inner-shell vacancy production.

Now, during the ion-atom collisions the nucleus of the target atom/projectile ion receives an impact, which gives it a recoil velocity $v$. If this perturbative impact is sudden relative to the electron orbital periodical

TABLE I

Normalized non-relativistic hydrogenic wave functions corresponding to the first three shells $K$-, $L$ - and $M$-shell electrons $[19,20]$. The spectroscopic notations have their usual meanings.

\begin{tabular}{c|c|c|c|c|c}
\hline \hline Shell & $n$ & $l$ & $m$ & $\begin{array}{c}\text { Orbital } \\
\text { designation }\end{array}$ & Wave function $\psi_{n l m}(r, \theta, \phi)$ \\
\hline$K$ & 1 & 0 & 0 & $1 s$ & $\frac{1}{\sqrt{\pi}}\left(\frac{Z}{a}\right)^{3 / 2} \exp \left(-\frac{Z r}{a}\right)$ \\
$L$ & 2 & 0 & 0 & $2 s$ & $\frac{1}{2 \sqrt{2 \pi}}\left(\frac{Z}{a}\right)^{3 / 2}\left(1-\frac{Z r}{2 a}\right) \exp \left(-\frac{Z r}{2 a}\right)$ \\
& 2 & 1 & 0 & $2 p_{z}$ & $\frac{1}{4 \sqrt{2 \pi}}\left(\frac{Z}{a}\right)^{3 / 2} \frac{Z r}{a} \exp \left(-\frac{Z r}{2 a}\right) \cos \theta$ \\
& 2 & 1 & -1 & $2 p_{y}$ & $\frac{1}{4 \sqrt{2 \pi}}\left(\frac{Z}{a}\right)^{3 / 2} \frac{Z r}{a} \exp \left(-\frac{Z r}{2 a}\right) \sin \theta \sin \phi$ \\
& 2 & 1 & 1 & $2 p_{x}$ & $\frac{1}{4 \sqrt{2 \pi}}\left(\frac{Z}{a}\right)^{3 / 2} \frac{Z r}{a} \exp \left(-\frac{Z r}{2 a}\right) \sin \theta \cos \phi$ \\
& 3 & 0 & 0 & $3 s$ & $\frac{1}{3 \sqrt{3 \pi}}\left(\frac{Z}{a}\right)^{3 / 2}\left(\frac{2 Z^{2} r^{2}}{27 a^{2}}-\frac{2 Z r}{3 a}+1\right) \exp \left(-\frac{Z r}{3 a}\right)$ \\
& 3 & 1 & 0 & $3 p_{z}$ & $\frac{2 \sqrt{2}}{27 \sqrt{\pi}}\left(\frac{Z}{a}\right)^{3 / 2} \frac{Z_{r}}{a}\left(1-\frac{Z r}{6 a}\right) \exp \left(-\frac{Z r}{3 a}\right) \cos \theta$ \\
& 3 & 1 & -1 & $3 p_{y}$ & $\frac{2 \sqrt{2}}{27 \sqrt{\pi}}\left(\frac{Z}{a}\right)^{3 / 2} \frac{Z r}{a}\left(1-\frac{Z r}{6 a}\right) \exp \left(-\frac{Z r}{3 a}\right) \sin \theta \sin \phi$ \\
& 3 & 1 & 1 & $3 p_{x}$ & $\frac{2 \sqrt{2}}{27 \sqrt{\pi}}\left(\frac{Z}{a}\right)^{3 / 2} \frac{Z r}{a}\left(1-\frac{Z r}{6 a}\right) \exp \left(-\frac{Z r}{3 a}\right) \sin \theta \cos \phi$ \\
& 3 & 2 & 0 & $3 d_{z^{2}}$ & $\frac{1}{81 \sqrt{6 \pi}}\left(\frac{Z}{a}\right)^{3 / 2}\left(3 \cos ^{2} \theta-1\right)\left(\frac{Z^{2} r^{2}}{a^{2}}\right) \exp \left(-\frac{Z r}{3 a}\right)$ \\
& 3 & 2 & -1 & $3 d_{y z}$ & $\frac{\sqrt{2}}{81 \sqrt{\pi}}\left(\frac{Z}{a}\right)^{3 / 2}\left(\frac{Z^{2} r^{2}}{a^{2}}\right) \exp \left(-\frac{Z r}{3 a}\right) \sin \theta \cos \theta \sin \phi$ \\
& 3 & 2 & 1 & $3 d_{x z}$ & $\frac{\sqrt{2}}{81 \sqrt{\pi}}\left(\frac{Z}{a}\right)^{3 / 2}\left(\frac{Z^{2} r^{2}}{a^{2}}\right) \exp \left(-\frac{Z r}{3 a}\right) \sin \theta \cos \theta \cos \phi$ \\
& 3 & 2 & -2 & $3 d_{x y}$ & $\frac{1}{81 \sqrt{2 \pi}}\left(\frac{Z}{a}\right)^{3 / 2}\left(\frac{Z^{2} r^{2}}{a^{2}}\right) \exp \left(-\frac{Z r}{3 a}\right) \sin { }^{2} \theta \sin 2 \phi$ \\
& 3 & 2 & 2 & $3 d_{x^{2}-y^{2}}$ & $\frac{1}{81 \sqrt{2 \pi}}\left(\frac{Z}{a}\right)^{3 / 2}\left(\frac{Z^{2} r^{2}}{a^{2}}\right) \exp \left(-\frac{Z r}{3 a}\right) \sin { }^{2} \theta \cos 2 \phi$
\end{tabular}

motion, it can lead to the excitation or ionization of the electrons [15], as discussed earlier. Now, let us first discuss sudden jolt in the target nuclei, assuming the lab frame of reference is $S$. After the ion-atom collision the frame of reference is $S^{\prime}$, which is moving with the nucleus. Due to the suddenness of perturbation, the coordinates of electrons remain same in $S^{\prime}$ as in the rest frame $S$.
The initial wave function of electron in $S^{\prime}$ is given by:

$$
\psi_{0}^{\prime}=\psi_{0} \exp \left(-\mathrm{i} \boldsymbol{q} \sum_{i} \boldsymbol{r}_{\beta}\right) .
$$

Here, $q\left(=\frac{m_{e}}{M_{T}+Z_{T} m_{e}} \frac{p}{\hbar}\right)$ is the wave-vector, $p$ is the recoiled target nucleus momentum in $S$ frame, $m_{e}$ and $M_{T}=$ electron and target nuclei mass respectively, 
$Z_{T}=$ target nuclei atomic number and $\psi_{0}$ is the wave function of the electron when the nucleus is at rest i.e. in the frame $S$. The summation is over all the $Z$ electrons in the corresponding atomic system.

From Eqs. (3) and (4), the required probability of electron to remain in the same state is given by:

$$
\begin{aligned}
& W=\left|\int \psi_{0}^{2} \exp (-\mathrm{i} \boldsymbol{q} \cdot \boldsymbol{r}) \mathrm{d} V\right|^{2}= \\
& \left|\int_{0}^{\infty} \int_{0}^{\pi} \int_{0}^{2 \pi} \psi_{0}^{2} \exp (-\mathrm{i} q r \cos \theta) r^{2} \sin \theta \mathrm{d} r \mathrm{~d} \theta \mathrm{d} \phi\right|^{2} .
\end{aligned}
$$

Similar formalism is also applicable for the projectile nuclei. Using non-relativistic hydrogenic wave functions $[19,20]$ given in Table I, we can get the probability of electron to remain in the same initial state after the sudden jolt of respective nuclei by the integration of Eq. (5). The results obtained are following:

$$
\begin{aligned}
& W_{100}^{K}=\frac{2^{8} Z^{8}}{\left(a^{2} q^{2}+4 Z^{2}\right)^{4}}, \\
& W_{200}^{L}=\frac{Z^{8}\left(2 a^{4} q^{4}-3 a^{2} q^{2} Z^{2}+Z^{4}\right)^{2}}{\left(a^{2} q^{2}+Z^{2}\right)^{8}}, \\
& W_{210}^{L}=\frac{Z^{12}\left(Z^{2}-5 a^{2} q^{2}\right)^{2}}{\left(a^{2} q^{2}+Z^{2}\right)^{8}}, \\
& W_{211}^{L}=W_{21-1}^{L}=\frac{Z^{12}}{\left(a^{2} q^{2}+Z^{2}\right)^{6}}, \\
& W_{300}^{M}=\left[2^{8} Z^{8}\left(3 a^{2} q^{2}-4 Z^{2}\right)^{2}\left(27 a^{2} q^{2}-4 Z^{2}\right)^{2}\right. \\
& \left.\times\left(243 a^{4} q^{4}-216 a^{2} q^{2} Z^{2}+16 Z^{4}\right)^{2}\right] / \\
& \left.W_{310}^{M}=\left[9 a^{2} q^{2}+4 Z^{2}\right)^{12}\right], \\
& \left.\left.\left.\quad+648 a^{2} q^{2} Z^{4}-16 Z^{6}\right)^{2}\right] /\left(9 a^{2} q^{2}+4 Z^{2}\right)^{12}\right] \\
& W_{321}^{M}=W_{32-1}^{M}=\frac{2^{16} Z^{14}\left(63 a^{2} q^{2} Z-4 Z^{3}\right)^{2}}{\left(9 a^{2} q^{2} q^{2}+4 Z^{2} q^{2}+4 Z^{2}\right)^{10}}, \\
& W_{311}^{M}=W_{31-1}^{M}=\frac{2^{16} Z^{14}\left(137 a^{4} q^{4} Z-312 a^{2} q^{2} Z^{3}+16 Z^{5}\right)^{2}}{\left(9 a^{2} q^{2}+4 Z^{2}\right)^{8}}, \\
& 2^{16} Z^{10}\left(81 a^{4} q^{4} Z-27 a^{2} q^{2} Z^{3}+4 Z^{5}\right)^{2}
\end{aligned}
$$

Here $W_{n l m}^{K}, W_{n l m}^{L}$ and $W_{n l m}^{M}$ represent the probability that the electrons will remain in the same shell i.e. $K$-, $L$ - and $M$-shell, respectively. Further, $a=$ Bohr radius and $Z=$ atomic number of the corresponding atomic system. For the case of hydrogen atom Eq. (6) reduces to the earlier reported value [15] i.e. $W_{100}^{K}=1 /\left(1+\frac{a^{2} q^{2}}{4}\right)^{4}$.

Thus, the shaking probability can be obtained by following relation:

$$
W_{\text {shaking }}=1-W_{\text {nlm }},
$$

Here, $W_{\text {nlm }}$ is the probability of electron to remain in the same initial state as defined in the Eqs. (6)-(15).

Thus, the shaking probability is defined by: where $W$ can be replaced by $W_{100}^{K}, W_{2 l m}^{L}$ or $W_{3 l m}^{M}$ from Eqs. (6)-

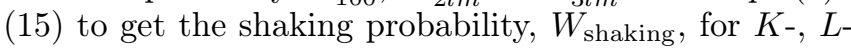
and $M$-shell electrons, respectively.

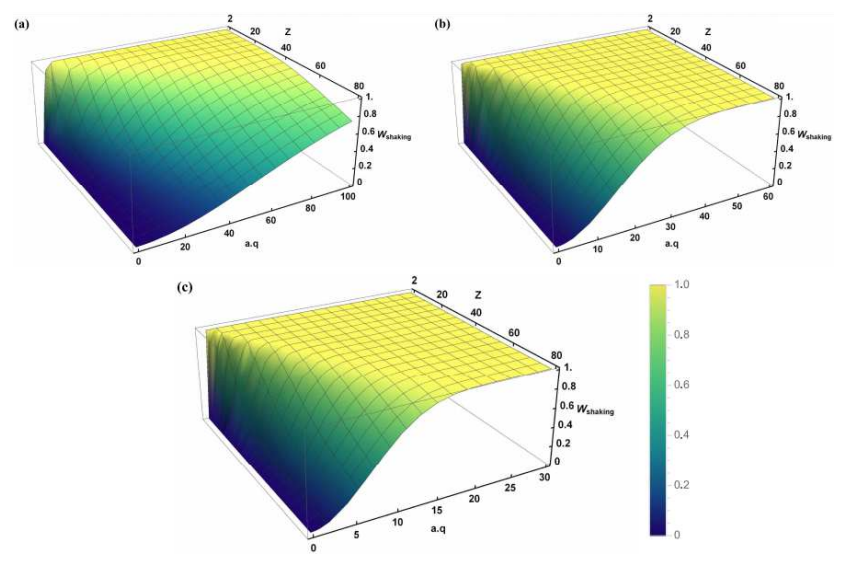

Fig. 1. Variation of shaking probability ( $W_{\text {shaking }}$ ) with respect to the $a q$ and atomic number $Z$ of the corresponding atomic system for the case of (a) $1 s$ subshell, (b) $2 s$ subshell, and (c) $3 s$ subshell.

It is found that for the values of $a q \ll 1$, the shaking probability reduces to zero whereas for the values of $a q \gg 1$ it tends to unity, which can clearly be seen in Fig. 1. One can further observe in Fig. 1 that the condition for total shaking $\left(W_{\text {shaking }}=1\right)$ can be achieved at comparatively lower values of $a q$ for outer shells than the inner shells. Interestingly during the fast ion-atom collisions, condition $a q \gg 1$ is applicable in a wide range of recoil energy (starting from $\mathrm{keV} / \mathrm{u}$ ). Noteworthy that applicability of the condition for sudden perturbation during the ion-atom collisions requires higher incident energy than the required recoil energy for initiation of the shaking process. It clearly suggests that once the sudden approximation condition is satisfied it will naturally assure the limiting condition $a q \gg 1$.

\section{Conclusion}

A discussion on the shaking process accompanying the ion-atom collisions is presented in the sudden approximation limit for hydrogen-like systems. Analytical expressions for the shaking probabilities have been derived for the $K-, L$ - and $M$-shell electrons using the nonrelativistic hydrogenic wave functions. It is found that in the limiting case i.e. $a q \ll 1$, the shaking probability reduces to zero, whereas for $a q \gg 1$ it tends to unity. Interestingly, the condition $a q \gg 1$ holds good even for the low recoil cases (starting from $\mathrm{keV} / \mathrm{u}$ ). During the fast ion-atom collision suddenness of impact can satisfy this 
condition very well. Thus, this study implies that during the fast heavy ion-atom collisions, one of the most probable channels of electron transitions is the shaking process, which occurs due to the sudden recoil of the projectile/target nuclei.

\section{Acknowledgments}

Prashant Sharma is thankful to UGC, India for providing the fellowship as financial support to carry out this work.

\section{References}

[1] J. Mcguire, Electron Correlation Dynamics in Atomic Collisions, Cambridge University Press, Cambridge 1997.

[2] M.S. Freedman, Ann. Rev. Nucl. Sci. 24, 209 (1974).

[3] K. Hecht, in: Quantum Mechanics, Graduate Texts in Contemporary Physics, Springer, New York 2000, p. 561.

[4] O.I. Feoktistov, Ukr. J. Phys. 55, 165 (2010).

[5] J.S. Levinger, Phys. Rev. 90, 11 (1953).

[6] C. Couratin, P. Velten, X. Fléchard, E. Liénard, G. Ban, A. Cassimi, P. Delahaye, D. Durand, D. Hennecart, F. Mauger, A. Méry, O. Naviliat-Cuncic, Z. Patyk, D. Rodríguez, K. Siegień-Iwaniuk, J.-C. Thomas, Phys. Rev. Lett. 108, 243201 (2012).
[7] P. Sharma, T. Nandi, Nucl. Phys. A 941, 265 (2015).

[8] H.J. Cho, S.K. Nha, J. Kor. Phys. Soc. 30, 521 (1997).

[9] T. Mukoyama, S. Shimizu, Phys. Rev. C 11, 1353 (1975).

[10] F.T. Porter, M.S. Freedman, F. Wagner, Phys. Rev. C 3, 2246 (1971).

[11] H.-J. Cho, S.-K. Nha, G.-D. Kim, J. Kor. Phys. Soc. 30, 180 (1997).

[12] A. Suzuki, J. Law, Phys. Rev. C 25, 2722 (1982).

[13] T. Mukoyama, X-Ray Spectrom. 39, 142 (2010).

[14] T. Mukoyama, X-Ray Spectrom. 34, 64 (2005).

[15] L. Landau, E. Lifshitz, Quantum Mechanics - Nonrelativistic Theory, Vol. 1, Pergamon Press, New York 1980.

[16] N. Zettili, Quantum Mechanics Concepts and Applications, 2nd ed., Wiley, 2009.

[17] A. Bohm, Quantum Mechanics, Springer, New York 1979.

[18] T.A. Carlson, J. Chem. Phys. 48, 5191 (1968).

[19] B.H. Bransden, C.J. Joachain, Physics of Atoms and Molecules, Longman, London 1983.

[20] Y. Mido, S.A. Iqbal, Atomic Structure, Discovery Publishing House, New Delhi 1991. 